\title{
Space Weather, Earth's Neutral Upper Atmosphere (Thermosphere), and Spacecraft Orbital Lifetime/Dynamics
}

\author{
Jerry K. Owens, William W. Vaughan, Keith O. Niehuss, and Joseph Minow
}

\begin{abstract}
Accurate prediction of a spacecraft/satellite orbital lifetime, insertion altitude, reboost requirements, and mission performance is mainly the result of the integrated effect from knowledge of the atmospheric density, space weather (solar/geomagnetic), and timeline of vehicle characteristics. Each of these elements is dependent upon a model developed to provide the inputs necessary for the use of an orbital lifetime prediction program. This paper will address relative influences of these elements with emphasis on solar/geomagnetic activity, atmospheric density, and drag coefficient associated with the model products used to predict orbital lifetime and related spacecraft/satellite design and operational conditions. Issues associated with the potential for improvement of the lifetime prediction model input elements will be discussed with regard to their relative contributions to improving orbital lifetime and performance predictions.
\end{abstract}

Index Terms-Atmosphere (thermosphere): structure/dynamics, modeling, solar activity/cycle variations, space weather, spacecraft interactions.

\section{INTRODUCTION}

$\mathbf{U}$ NCERTAINTIES in spacecraft orbital lifetime prediction are due mainly to estimated future solar and geomagnetic activity inputs, modeled atmospheric density, and predicted ballistic factor. Fig. 1 illustrates these elements. The major source of uncertainty in models estimating future atmospheric densities at orbital altitude is the solar extreme ultraviolet heat input values. The observed 10.7-cm solar radio flux (not adjusted to $1 \mathrm{AU}$ ) is used as a proxy for this most significant input, which is not otherwise available.

A statistical model was developed to estimate the future 13-month Zurich smoothed monthly solar flux values. The model was structured to provide the detail needed for input to spacecraft/satellite orbital lifetime prediction models. Included in the model is the capability to provide monthly updates of future solar activity estimates. The performance of the model and current results are described in this paper.

Because no generally accepted physical solar model is available to accurately predict future solar activity, the NASA Mar-

\footnotetext{
Manuscript received December 4, 1999; revised September 19, 2000. This paper was presented in part at the American Astronomical Society/American Institute of Aeronautics and Astronautics Astrodynamics Specialists Meeting, Girdwood, AK, August 16-19, 1999.

J. K. Owens, and K. O. Niehuss are with the NASA Marshall Space Flight Center, Huntsville, AL 35812 USA.

W. W. Vaughan is with the University of Alabama, Huntsville, AL 35899 USA.

J. Minow is with the Sverdrup Technology, Inc., Huntsville, AL 35806 USA. Publisher Item Identifier S 0093-3813(00)11342-6.
}

shall Space Flight Center (MSFC) developed a Lagrangianbased 13-month smoothed solar flux and geomagnetic index intermediate (months) and long-range (years) statistical estimation technique [1]. The reason for issuing intermediate and long-range solar activity (solar flux and geomagnetic activity) estimates is the need for updated inputs to the upper atmosphere density models used for satellite orbital lifetime predictions and performance requirement analyzes [2]. Mission analysis and planning for future spacecraft launches and on-orbit operations require estimates of orbital lifetimes, altitudes, inclinations, and eccentricities.

Future estimates of ambient total density for computing atmospheric drag (the major perturbation for orbiting spacecraft) is of great importance for satellite dynamics and orbit predictions. A number of issues are affected, including lifetime estimates, orbit determination and tracking, attitude dynamics, and reentry prediction. These issues further affect logistics planning through attitude control requirements and reboost planning. The neutral thermosphere is important for two reasons. First, even at its low density, it produces significant torques and drag on orbiting spacecraft and orbital debris. Second, the density-height profile of the atmosphere above $100 \mathrm{~km}$ altitude modulates the flux of trapped radiation encountered at orbital altitudes. The Marshall engineering thermosphere (MET) model of the earth's atmosphere at spacecraft orbital altitudes evolved, based on work conducted at the NASA Marshall Space Flight Center (MSFC), over a long period of time [3]. The model is based on the extensive work of Luigi Jacchia and his colleagues at the Smithsonian Astrophysical Observatory during the 1960s and 1970s.

\section{NASA MARSHALl SOLAR ACTIVITY FUTURE ESTIMATION MODEL}

Vitinskii [4] conducted an extensive survey and analysis of solar activity prediction methods. While recognizing the magnitude of the problem and encouraging studies of active processes taking place on the Sun to solve it, he reiterated what still remains the current status [5]-[7]: “ $\ldots$ we have shown that the reliability of the results obtained using these methods still leaves much to be desired." His analysis, however, showed the linear regression method usually gives relatively accurate results to about a year in advance. For several-years-in-advance, the linear regression method becomes increasingly less accurate.

McNish and Lincoln [8] suggested that the estimation of a sunspot cycle's future behavior, based on the mean approxima- 


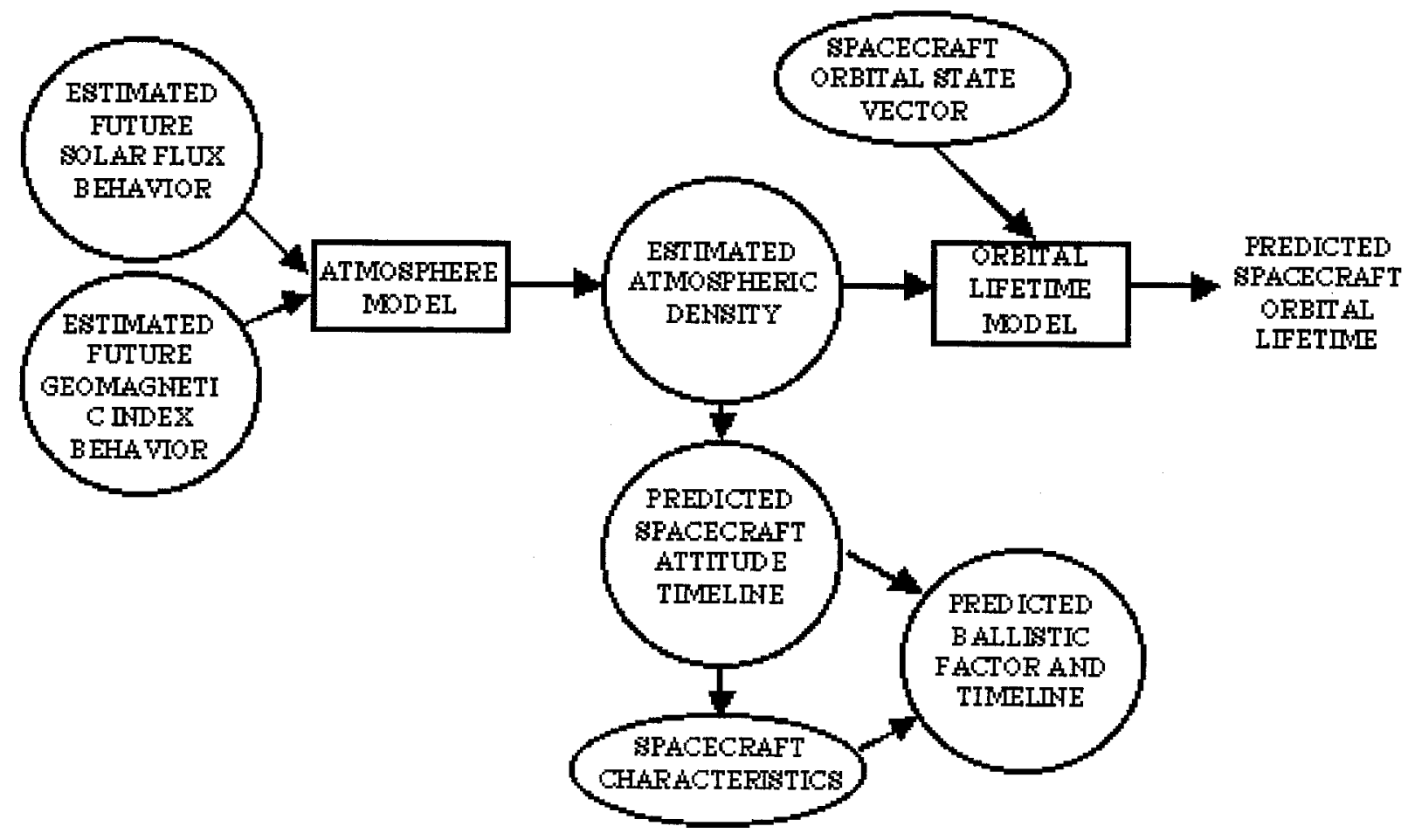

Fig. 1. Spacecraft orbital lifetime prediction technique block diagram.

tion of all past cycles, could be improved by adding to the mean a correction proportional to the departure of the current value of the cycle from the mean cycle. They also recommended the method not be used for making future projections longer than one year. Using a database with two additional solar cycles, Boykin and Richards [9] modified the McNish-Lincoln linear regression method so the 13-month Zurich smoothed relative sunspot number could be estimated for ten years in advance, at quarterly rather than yearly intervals. This method is also applicable to 13 -month smoothed solar $10.7 \mathrm{~cm}$ radio flux $\left(\bar{F}_{10.7}\right)$ and to some degree the geomagnetic index $\left(\bar{A}_{p}\right)$.

Holland and Vaughan [10] determined that better statistical estimations are possible, in a chi square sense, by selecting the start and end of each set (solar cycle) at the maximum (or minimum) and normalizing the data sets using a Lagrangian linear regression statistical technique. This determination and initialization of the modified McNish-Lincoln linear regression method at the cycle's maximum or minimum constitute the current NASA Marshall solar activity future estimation (MSAFE) model for intermediate and long-range estimation of the 13-month Zurich smoothed solar radio flux and geomagnetic index.

Although some researchers believe they have sufficient reason to separate the data for sunspot cycles 1 through 8 from the total database, the MSAFE model uses the observed data for all observed cycles. Including cycles 1 through 8 provides information applicable to the apparent behavior of the cycle period and to the overall magnitude during this time frame as well as a larger database for statistical estimates. The 13-month smoothed measured solar $10.7-\mathrm{cm}$ radio flux database was extended back to 1749 by using Wolf's relative sunspot values and a conversion equation based on solar flux and sunspot number observations from 1947 to 1995 , both smoothed using the 13-month Zurich smoothing technique.

The MSAFE model is reinitialized when a new minimum or maximum has been identified in the observed (not corrected to $1 \mathrm{AU})$ 13-month Zurich smoothed solar flux measurements. This reinitialization of the model provides for a more accurate estimation of the future smoothed solar radio flux. The early identification of the occurrence of a new minimum or maximum is thus important relative to providing timely, accurate outputs of future solar activity estimates by the model.

By screening the available solar flux measurements since 1947, criteria were developed for early identification of the new cycle minimum and maximum [11]. Fig. 2 illustrates the application of these criteria. This logic was used to identify the minimum for solar cycle 23 in the 13-month Zurich smoothed solar radio flux measurements. It provided a provisional identification of the May 1996 minimum for cycle 23 at 71.4 flux units when the June 1996 13-month Zurich smoothed solar flux value of 71.8 was observed (once the December 1996 monthly mean solar flux data were available). When the smoothed solar flux value exceeded 76 units for April 1997 (76.8 units), the May minimum date for Cycle 23 was confirmed based on the criteria previously developed.

\section{MSAFE Model Evaluation, CurRent Results, AND ENGINEERING USE}

Fig. 3 illustrates the performance of the MSAFE model for cycles 20 and 21 . The diagrams on the top are for initialization of the model at solar cycle minimum; those on the bottom are 


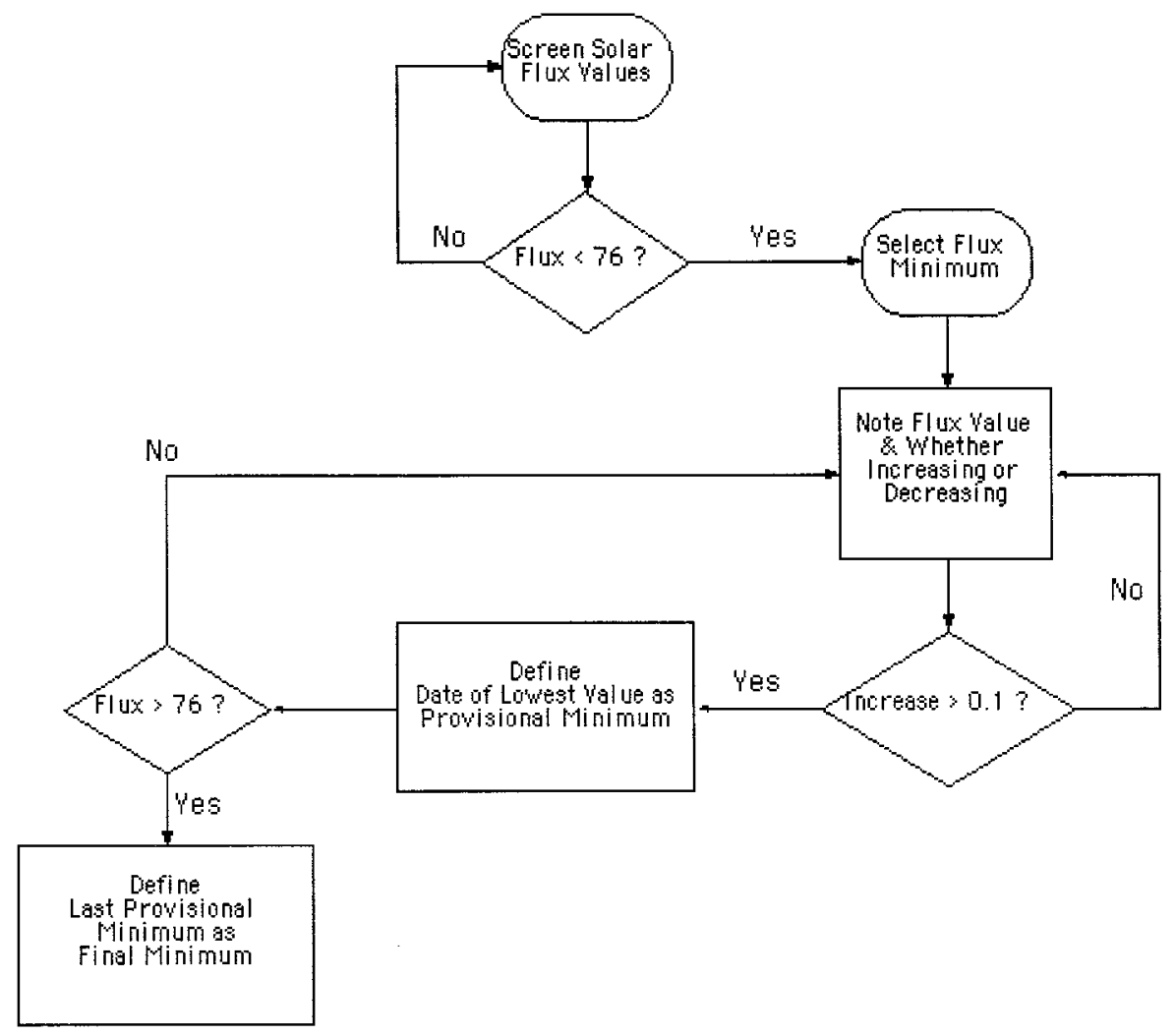

Fig. 2. Solar cycle minimum date selection logic.

for initialization of the model at maximum. As can be seen, the farther out in time the model estimates are from the initialization date, the less reliable the results relative to the confidence bounds shown. This is the reason the model is reinitialized each time a new minimum or maximum is determined. This problem is also mitigated by the monthly updates, which use all solar flux data up to the current month. The updates then provide a new set of future solar activity estimates starting with data from the current month.

For use of the MSAFE model output in atmospheric density models, the monthly update of the model output provides a timely input of future solar activity estimates to thermospheric density models, e.g., the NASA Marshall engineering thermosphere (MET) model. This enables the products of an orbital lifetime prediction model to be based on the latest measurements of the current solar cycle's solar radio flux through the MSAFE model output.

Fig. 4 provides the August 1999 output of the MSAFE model for the balance of cycle 23 and cycle 24 . It should be noted that the cycle 24 values are the statistical evaluation of the past 22 cycles and are not influenced by the MSAFE model's performance. Once the model is reinitialized to the maximum of cycle 23, it will provide estimates of future solar activity to the maximum of cycle 24. Long-range statistical estimates extending into cycle 24 are required for some orbital lifetime prediction activities. This is of particular importance for the early analysis of performance expectations for long lifetime spacecraft/satellites. Of concern is the estimation of reboost requirements for system design and orbital insertion altitude selections.

Since there is no method for intermediate or long-term predictions of daily $F_{10.7}$ or $a_{p}$, orbital lifetime predictions use the 13-month Zurich smoothed estimates [2]. Orbital lifetime predictions, control analysis programs, etc., require a specific date to associate with the future estimate of solar activity to compute corresponding atmospheric density. MSAFE model future estimated smoothed solar flux and geomagnetic index data points should be identified with the first day of the given month.

For spacecraft projects requiring a minimum risk design lifetime orbital altitude(s) and/or a specified control capability, the 95 percentile estimates of smoothed solar flux and geomagnetic index are recommended. Taking into account the short-term (days) dynamics, these estimates permit the design of a statistically conservative spacecraft lifetime and control capability for a given orbital altitude(s). The lifetime determination should be based on the most current intermediate and long-range statistical estimate of the future solar flux and geomagnetic index consistent with the critical project development decision time points prior to planned launch of the spacecraft.

Changes in orbital density associated with short-term variations in the daily $F_{10.7}$ and $a_{p}$ required as inputs to the upper atmospheric models such as the MET-99, 1999 version [3], are not represented by the 13-month Zurich smoothed solar flux and geomagnetic index statistical estimates given by the MSAFE model. Future changes in total atmospheric density cannot be estimated with any acceptable degree of statistical 

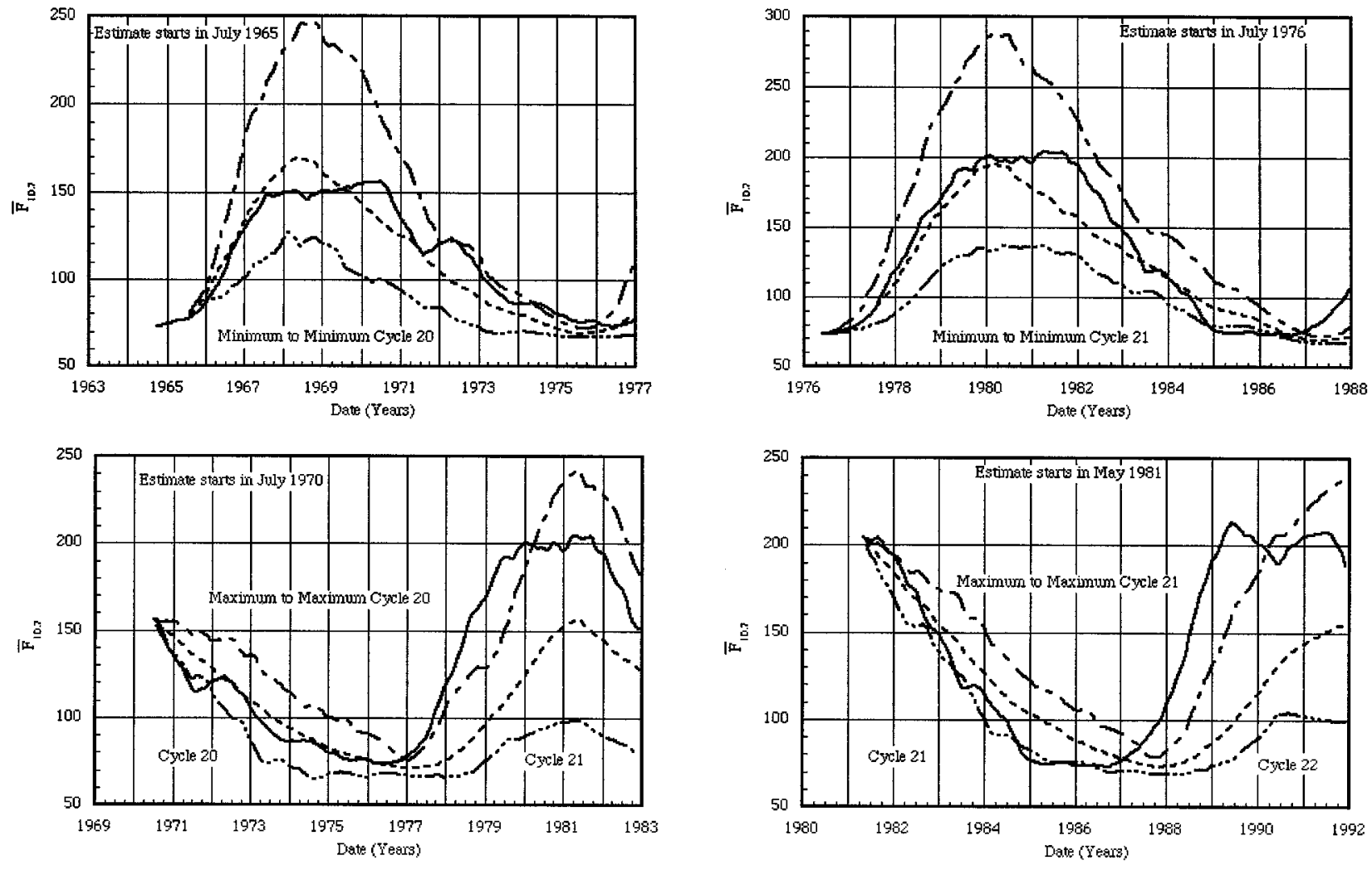

$\overline{--}-=$ Denotes gs of Perceritile Vabus

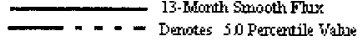

Fig. 3. NASA Marshall solar activity future estimation model results evaluation.

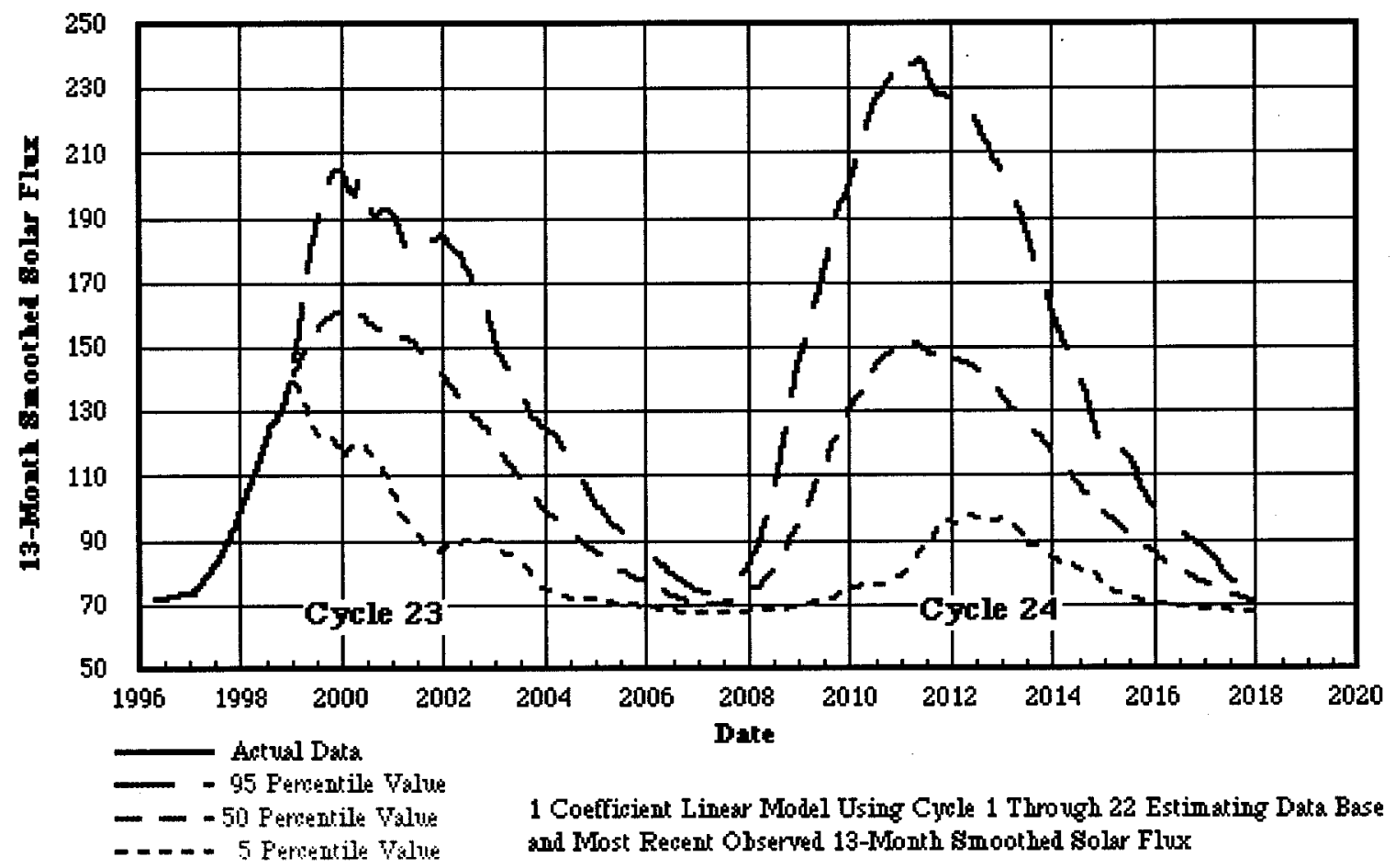

Fig. 4. NASA Marshall solar activity future estimation model current results.

confidence using existing techniques. Representative data sets, based on past daily $F_{10.7}$ and $a_{p}$ values, may be utilized to compute this dynamic component of the orbital altitude density. 


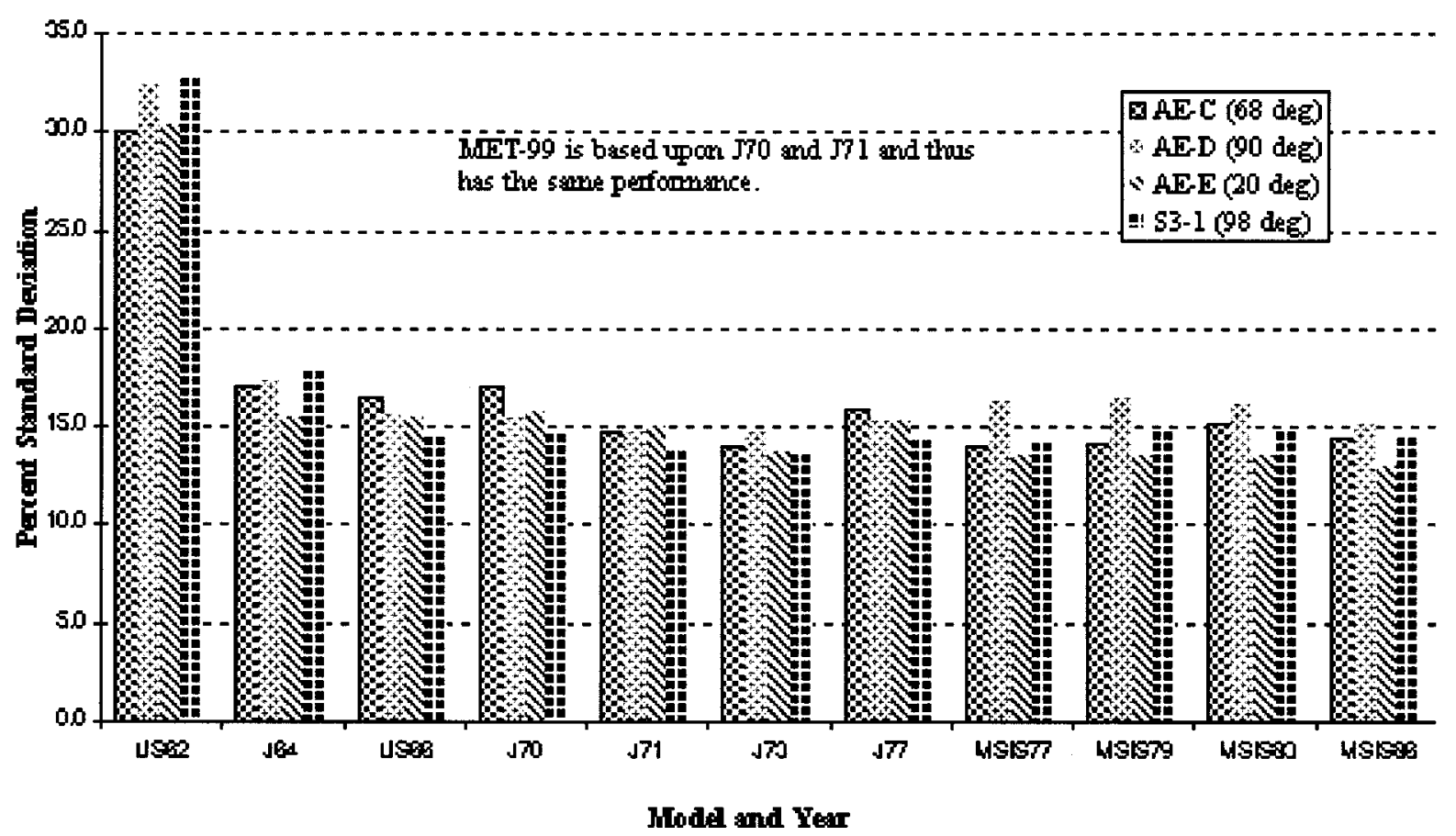

Fig. 5. Statistical evaluation of thermospheric neutral density models. (Note: The MET-99 model reflects the J70/J71 model's accuracy results, after Marcos et al. [15]).

\section{NASA MARShALl ENGINEERING THERMOSPHERE MODEL-1999 VERSION}

The MET-99 is a semi-empirical model using the static diffusion method with coefficients obtained from satellite drag analyzes [3], [12]. It is based on the NASA Marshall engineering thermosphere model, 1988 version [13], [14], developed from the Jacchia series of models. With the proper input parameters, specified below, an approximate exospheric temperature can be calculated. With exospheric temperature specified, the temperature can be calculated for any altitude between 90 and 2500 $\mathrm{km}$ from an empirically determined temperature profile. In the original development phase of the model the prime objective was to model the total neutral mass density of the thermosphere by adjusting temperature profiles until agreement between modeled and measured total densities was achieved. Agreement between modeled temperatures and temperatures measured on later missions was not always achieved. Thomson-scatter radar temperature measurements generally show that the diurnal temperature maximum lags the density maximum by a couple of hours, whereas in the MET- 99 model the temperature and the density maxima and minimal are in phase.

Studies of the accuracy with which thermospheric models estimate the neutral density have shown that an apparent "barrier" exists at the $15 \%$ standard deviation level, and models have not thus far been able to achieve better performance. A recent study on this subject by Marcos et al. [15] has shown that, historically, this level of accuracy was first achieved by Jacchia's 1970 and 1971 models. Fig. 5 illustrates this point. The NASA MET model [3] is based on the 1970 and 1971 Jacchia models and thus has the same $15 \%$ standard deviation performance. The MET model is used extensively as an input to satellite orbital lifetime and dynamics prediction models. The NASA MSAFE
TABLE I

THERMOSPHERIC DRIVERS INCLUDED IN MET-99

\begin{tabular}{c|c|c}
\hline $\begin{array}{c}\text { Effects Driving } \\
\text { the } \\
\text { Thermosphere }\end{array}$ & $\begin{array}{c}\text { Time } \\
\text { scale of } \\
\text { the Effect }\end{array}$ & $\begin{array}{c}\text { Is the Effect } \\
\text { Modeled by } \\
\text { MET-99 }\end{array}$ \\
\hline $\begin{array}{c}\text { Flux (Solar } \\
\text { Cycle) }\end{array}$ & Years & Yes \\
\hline Flux (Daily) & Day & Yes \\
\hline $\begin{array}{c}\text { Geomagnetic } \\
\text { Activity }\end{array}$ & Hours & Yes \\
\hline Local Time & Hours & Yes \\
\hline Semi-Annual & Months & Yes \\
\hline Latitude & Months & Yes \\
\hline Longitude & Day & Yes \\
\hline Waves & Minutes & Statistical Add-on \\
\hline
\end{tabular}

model [1] was developed specifically to provide inputs to the MET density model.

The essence of the MET-99 model is the calculation of atmospheric density in two major regions: the lower thermosphere (altitude $90 \mathrm{~km} \leq z \leq 105 \mathrm{~km}$ ) and the upper thermosphere $\left(z>10^{5} \mathrm{~km}\right)$. Between the base of the thermosphere (assumed here to be at $90 \mathrm{~km}$ ) and $105 \mathrm{~km}$, turbulent mixing is assumed to predominate, and diffusion dominates at higher altitudes. The density for all points on the globe at $90-\mathrm{km}$ altitude is assumed constant and mixing of atmospheric constituents prevails to $105 \mathrm{~km}$. Between these two altitudes the mean molecular mass varies as a result of dissociation of molecular oxygen to atomic oxygen. An empirical process is employed in the determination of the mean molecular mass distribution between 90 and 105 $\mathrm{km}$ such that the ratio of atomic to molecular oxygen is 1.5 at 


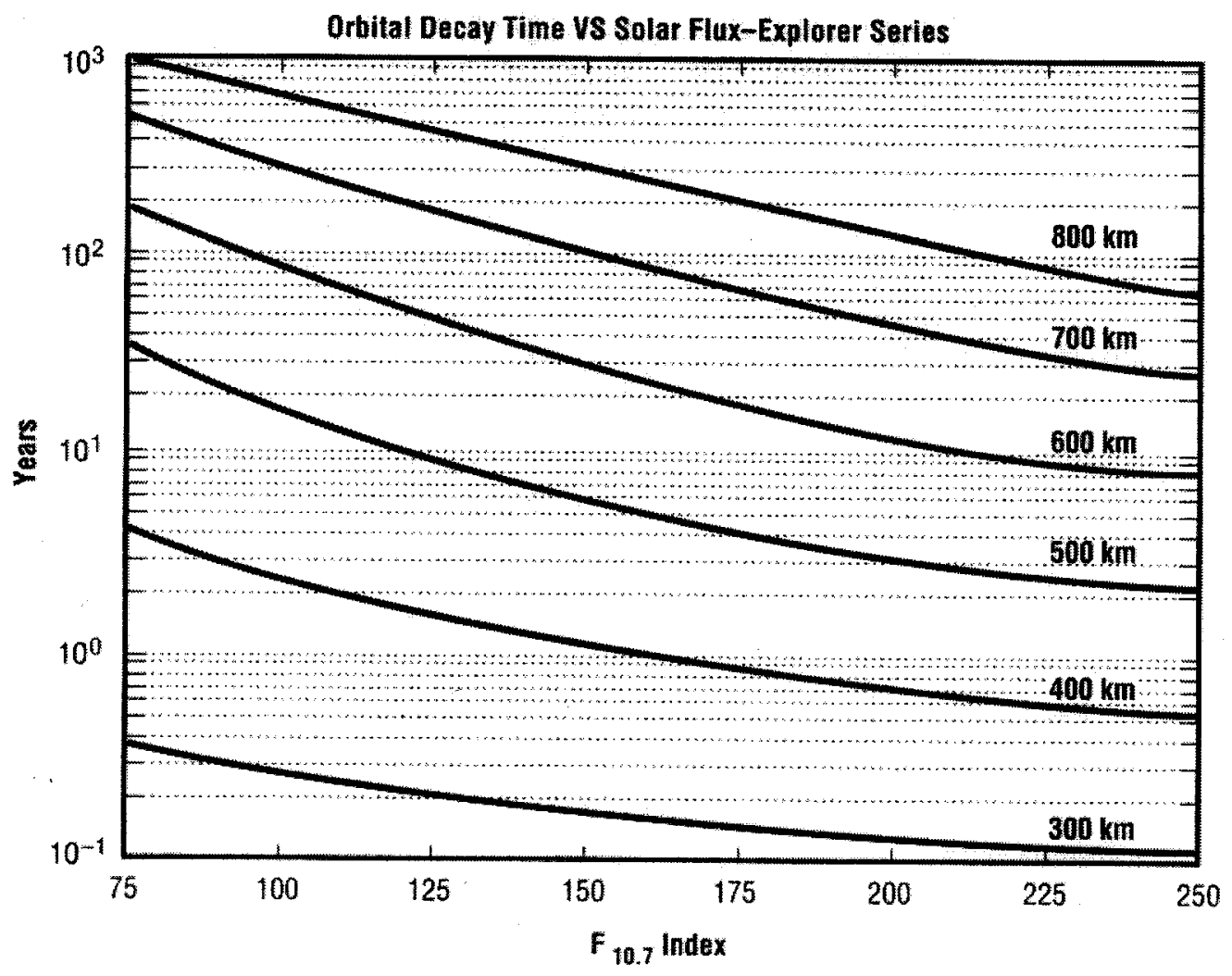

Fig. 6. Orbital decay time versus solar flux [18].

$120 \mathrm{~km}$. This makes it agree more closely with observations as reviewed by Von Zahn [16]. The input parameters required by the MET- 99 program are altitude, latitude, longitude, date (month, day, and year), time (hour and minute), three-hourly geomagnetic index (linear or logarithmic), and the observed daily $10.7-\mathrm{cm}$ solar radio flux and its average over six solar rotations referenced to the midpoint.

Table I illustrates the principal effects influencing the model's performance time scales. All of these effects, except those of short period atmospheric waves, are incorporated into the formulation of the MET-99 model. The effect of short term wave perturbations on the total density structure may be accounted for by a statistical add-on to the output of the MET-99 model.

\section{SOlar ACTIVITY, THERMOSPHERIC DENSITY, AND SATELLITE DRAG}

Fig. 6 provides an example of satellite lifetime as a function of solar radio flux for a satellite in circular orbits at various initial altitudes. The primary operational effect of upper atmospheric neutral density variability is on satellite drag. Short-term variations in total density, which occur during geomagnetic events, perturb the orbital motions of satellites. This leads to difficulties in tracking and cataloging objects in low earth orbit. These short-term perturbations also lead to uncertainties in position for reentry of orbiting vehicles. Long-term variations in atmospheric density, such as those driven by solar cycle variations in the extreme ultraviolet irradiance, have order-of-magnitude effects on the lifetime of satellites in low earth orbit.
Solar activity has a critical impact on most aspects of the ambient environment that a spacecraft experiences. Variations in solar activity impact the upper atmospheric (thermospheric) density levels, overall thermal environment, plasma density levels, meteoroid and orbital debris levels, flux and energy distribution of ionizing radiation, and characteristics of the earth's magnetic field. Through the influence of these aspects of the ambient space environment, the solar cycle also impacts mission planning and operation activities. For example, as can be noted from Fig. 6, when solar activity is high, the associated ultraviolet radiation from the sun heats and expands the earth's upper atmosphere. This increases atmospheric drag and, thus, the orbital decay rate of spacecraft.

\section{RATIONALE FOR ORbITAL LIFETIME PREDICTION ANALYSIS}

As noted in the previous sections, there are three principal elements that affect the prediction of a satellite's orbital lifetime (see Fig. 1). One is the ballistic factor, which is a function of the satellite's effective cross sectional area in the plane normal to the velocity vector, the satellite's mass, and the value of drag coefficient. The second is the ambient total atmospheric density at orbital altitude. The third is the estimation of future solar and geomagnetic activity used as an input to the density model.

The solar activity input most commonly used is the solar flux, $F_{10.7}$, as a surrogate for the EUV heating of atmosphere since the historical records and current measurements for EUV are rather limited. Also, the atmospheric density model was developed based on $F_{10.7}$ measurements. Thus, the prediction of a satellite's orbital lifetime, plus associated insertion altitude, reboost requirements, and mission performance, is mainly the re- 

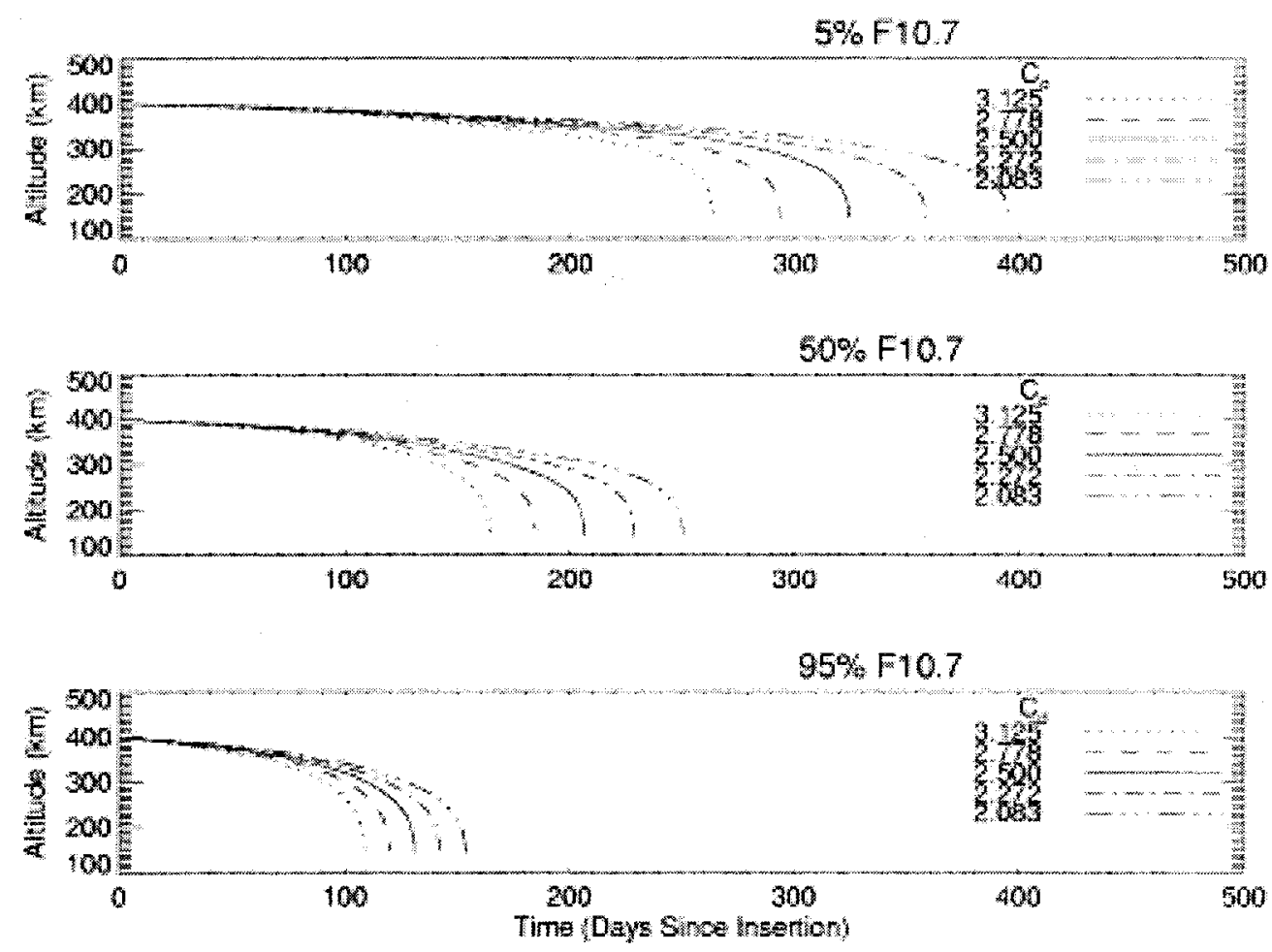

(a)

$\mathrm{Co}-2.500$

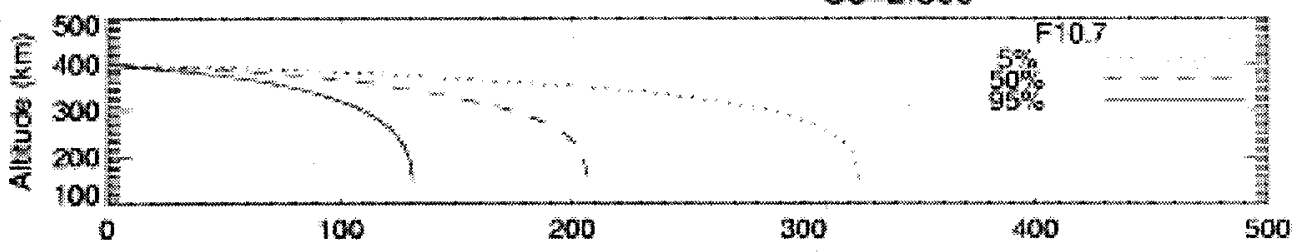

$\mathrm{Cd}=2.083$

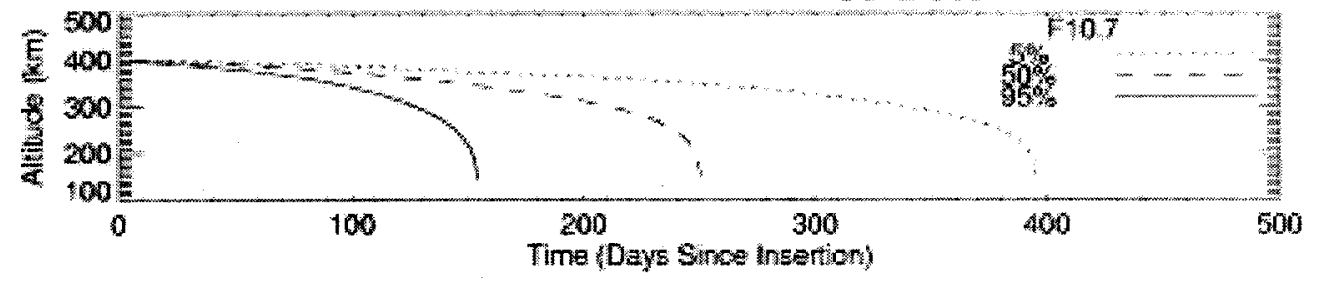

(b)

Fig. 7. Satellite decay from an initial altitude of $400 \mathrm{~km}$ : (a) $F_{10.7}$ estimates at the 5, 50, and $95 \%$ levels versus drag coefficient $\mathrm{Cd}$ and (b) $\mathrm{Cd}$ values of 3.125 , 2.5 , and 2.083 versus $F_{10.7}$ estimates at the 5, 50, and $95 \%$ levels.

sult of an integrated effect from knowledge of the atmospheric density, solar activity, and timeline of vehicle characteristics.

Much has been written on future solar activity, sunspots and $F_{10.7}$, model developments, and their relative performances, which covers a rather wide spectrum of results. Several studies have shown strong evidence that orbital altitude atmospheric density models represent the actual conditions with an uncertainty of about $15 \%$ standard deviation. This has been discussed in the section concerning the MET model development and performance. Until recently, the $15 \%$ standard deviation has 

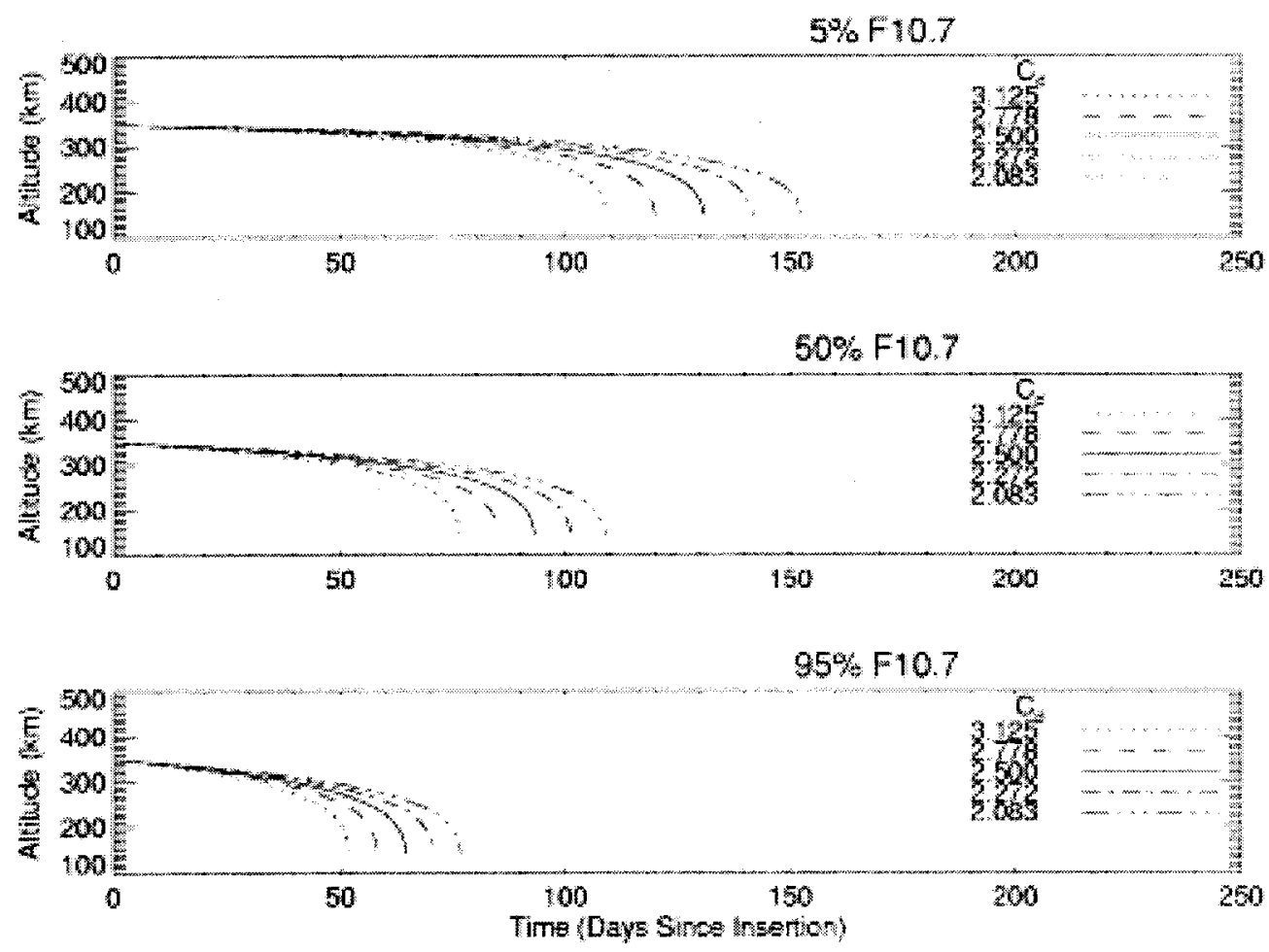

(a)
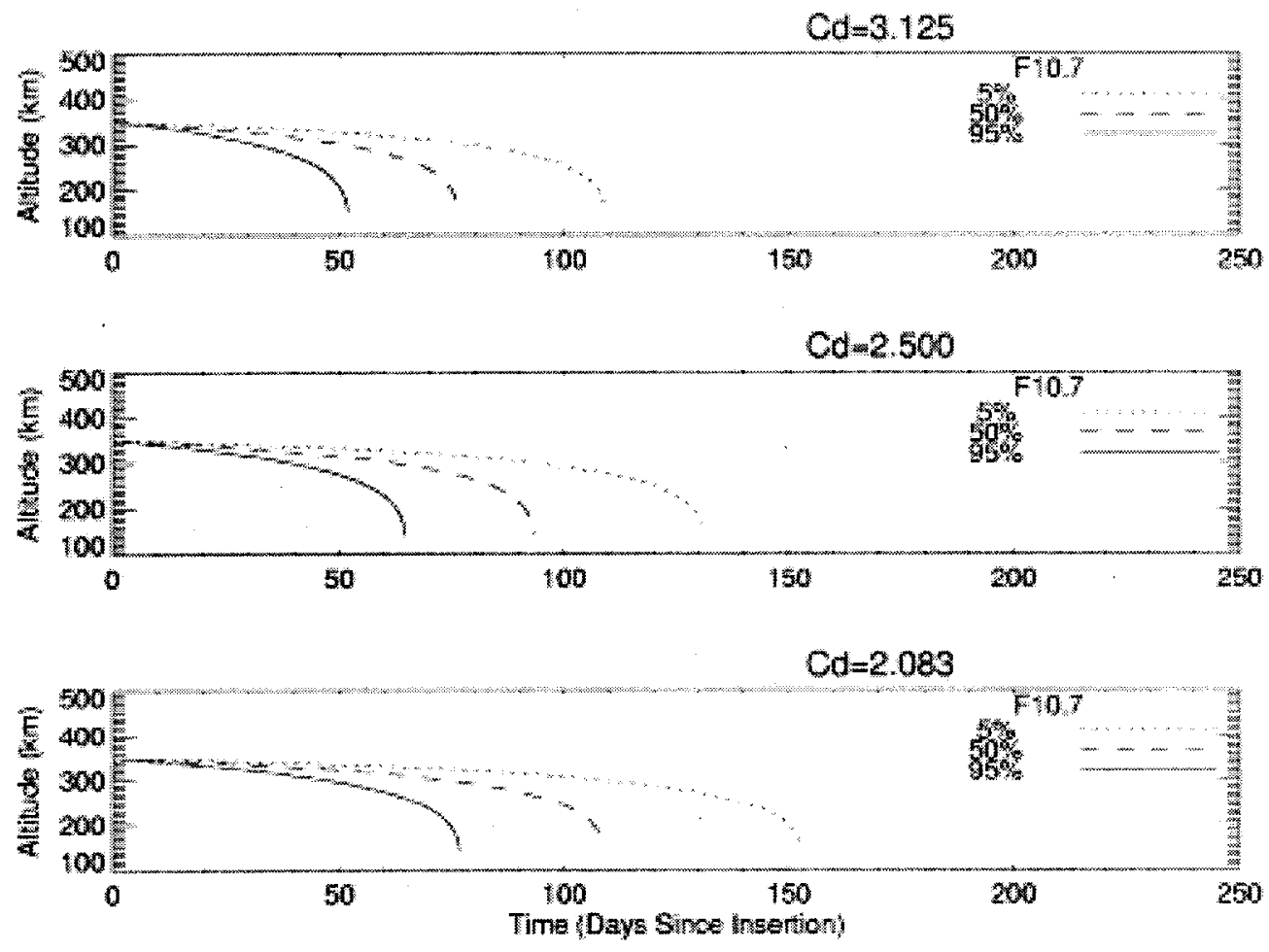

(b)

Fig. 8. Satellite decay from an initial altitude of $350 \mathrm{~km}$ : (a) $F_{10.7}$ estimates at the 5, 50, and $95 \%$ levels versus drag coefficient Cd and (b) Cd values of 3.125 , 2.5 , and 2.083 versus $F_{10.7}$ estimates at the 5,50 , and $95 \%$ levels.

appeared to be a limit on model performance, but recent analyzes indicate this uncertainty can be reduced by at least one-half. Recently, a data assimilation technique provided a capability to reduce these errors to the 5\% level [17]. As for the drag coefficient, this remains an area of some uncertain accuracy due to accommodation coefficient knowledge, etc.
Within the ballistic factor, while mass can be estimated or even measured rather well, the effective cross sectional area for nonspherical or noncylindrical satellites continues to be an area of some uncertainty to estimate in advance. Configurations such as the Skylab and most recently the relatively complex configuration represented by the International Space Station, 

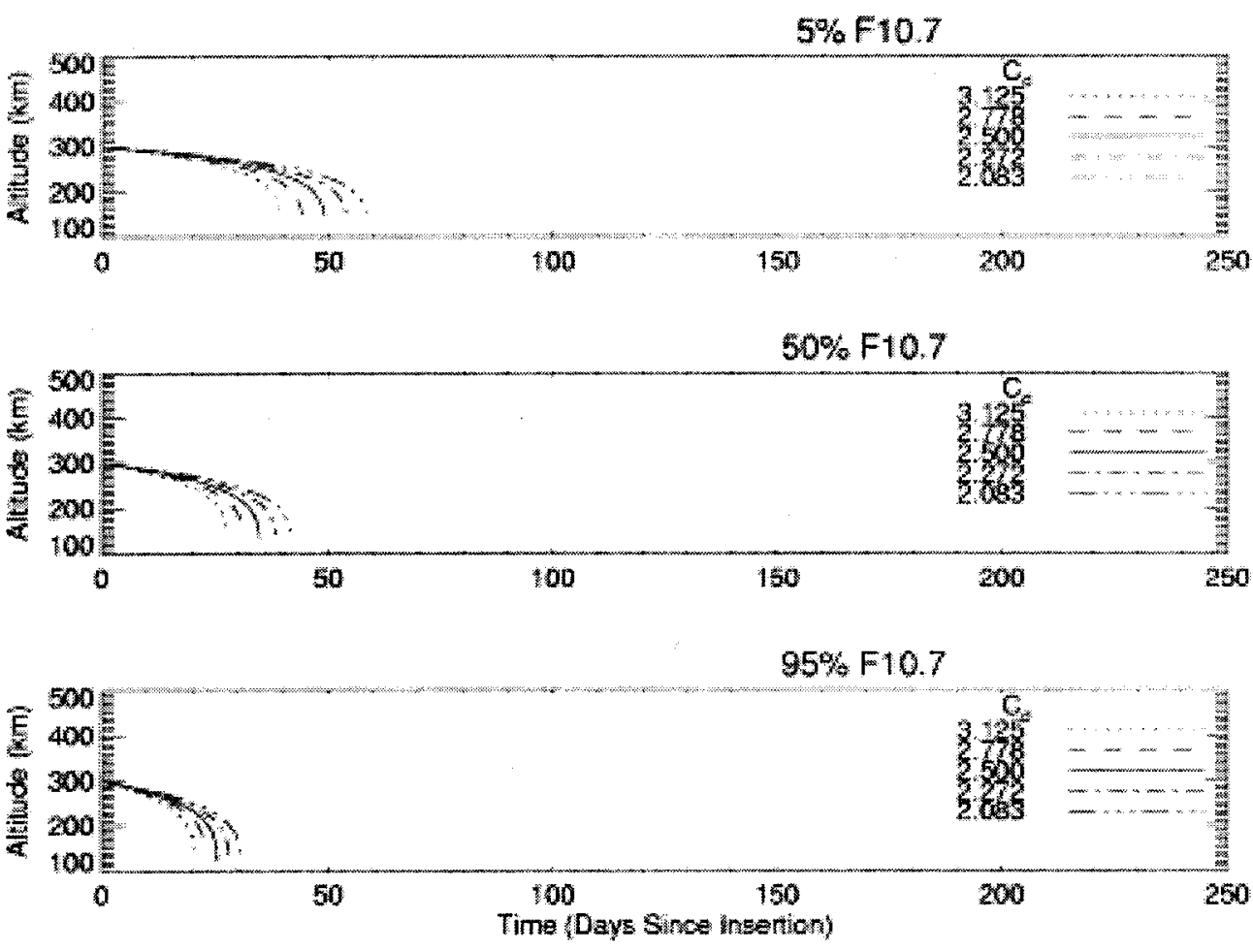

(a)
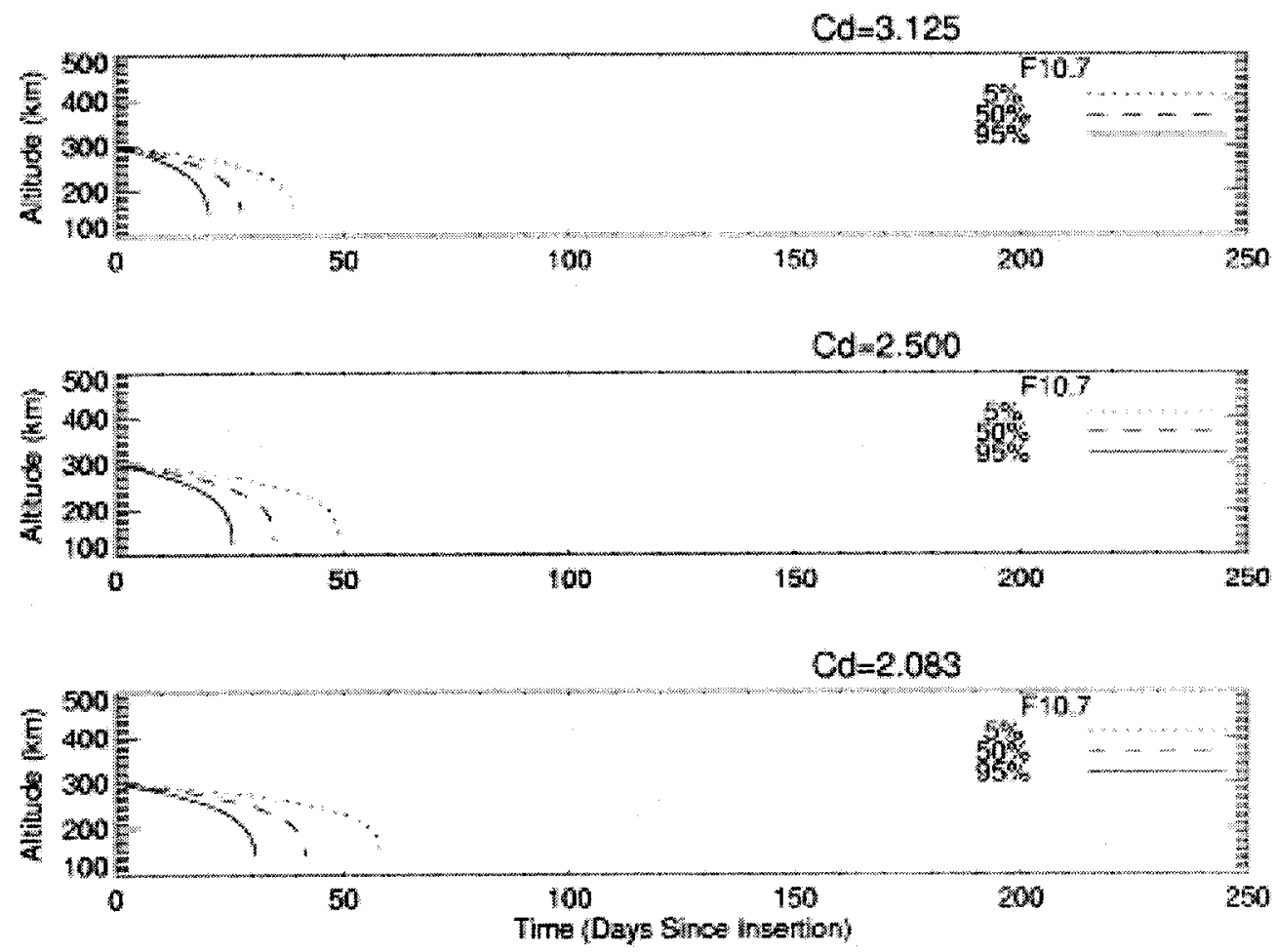

(b)

Fig. 9. Satellite decay from an initial altitude of $300 \mathrm{~km}$ : (a) $F_{10.7}$ estimates at the 5, 50, and $95 \%$ levels versus drag coefficient $\mathrm{Cd}$ and (b) $\mathrm{Cd}$ values of 3.125 , 2.5 , and 2.083 versus $F_{10.7}$ estimates at the 5,50 , and $95 \%$ levels.

relative to on-orbit effective cross sectional areas for use in the ballistic factor, continue to provide a challenge for accurate estimates. However, one element for orbital lifetime prediction models that has a major influence is the uncertainty in the estimate of future solar activity, which feeds into the atmospheric density model, and thus into the drag calculations. This determines the satellite performance/lifetime. This uncertainty has been discussed in the section concerning the MSAFE model development and performance.

The thrust of the analysis was to address the relative sensitivities of orbital lifetime predictions for a rather simple spherical satellite configuration initialized at three different orbital alti- 
tudes of $400 \mathrm{~km}, 350 \mathrm{~km}$, and $300 \mathrm{~km}$. The lifetime calculations were made over a range of drag coefficient variations for recent $5 / 50 / 95 \% F_{10.7}$ outputs of the MSAFE model. In turn, three representative drag coefficient values were used to calculate orbital lifetimes for the $5 / 50 / 95 \% F_{10.7}$ outputs of the MSAFE model. No variations were made in the satellite's effective cross sectional area or mass.

\section{RESUlts OF CAlCUlations}

The series of Figs. 7-9 provide a graphical product for the calculations. Fig. 7 is for the 400-km initial orbital altitude, Fig. 8 is for 350-km altitude, and Fig. 9 is for $300-\mathrm{km}$ altitude. If one compares the results of Figs. 7(a), 8(a), and 9(a), whereby the variations in $5 / 50 / 95 \% F_{10.7}$ MSAFE model products versus a range of drag coefficient values are presented, it is evident that the satellite's predicted lifetime can vary significantly, depending on the three elements of initial altitude, solar activity levels, and drag coefficient used. A similar spread in the predicted lifetime values for the satellite can be noted when one compares the results shown in Figs. 7(b), 8(b), and 9(b) where a variation in drag coefficients versus the 5/50/95\% MSAFE model output for $F_{10.7}$ is shown for the three initial orbital altitudes of $400 \mathrm{~km}, 350 \mathrm{~km}$, and $300 \mathrm{~km}$. These figures, in particular, illustrate the sensitivity of the predicted satellite lifetime to the estimated future solar activity as reflected by the $5 / 50 / 95 \%$ levels for the MSAFE model products. Studying these three figures provides one with considerable insight on the possible variations in orbital lifetime predictions that may result from uncertainties in the element values used in the lifetime prediction model. In particular, they illustrate the necessity to use timely outputs of model products for estimates of future solar activity such as those given by the monthly MSAFE model, and conservative 95\% level for future mission performance and planning decisions.

One may also note the significant effect on orbital lifetime by change in the initial orbital altitude from $400 \mathrm{~km}$ (Fig. 7) to $300 \mathrm{~km}$ (Fig. 9). Of course, increasing the orbital altitude above $400 \mathrm{~km}$ rapidly increases the satellite's lifetime relative to atmospheric density and thus minimizes the sensitivity to solar activity variations. A similar effect can be noted from Figs. 7(b) and 9(b) relative to the change in initial orbit altitude for the satellite-versus-drag coefficient variation.

The results of the calculations illustrate the relative sensitivities of a satellite in low earth orbit (LEO) to future solar activity inputs and variations in drag coefficient estimates [18]. It is readily apparent that the uncertainties associated with our current talent for estimating future solar activity significantly out weights the sensitivity due to even large errors in drag coefficient estimation. Not only is this an important observation illustrated graphically for the calculations, but further illustrates the importance of coordinated and consistent timely updates of estimated for future solar activity as provided monthly by the MSAFE model and use of a conservative 95\% level input for mission planning and design.

\section{CONCLUSION}

"State-of-the-art" in orbital altitude atmospheric density models performance of about $15 \%$ standard deviation does not appear to be the plateau previously indicated. However, space weather plays a significant role in the application of these density models and estimates from the various future solar activity prediction models, which still do not produce consistent results. Statistical techniques remain the major basis for such models. Maintaining coordinated, consistent, and timely solar activity model product outputs is important for use with conservative inputs in satellite mission planning and design decisions. One must remember that the orbital lifetime prediction process is an integrated process. Each element must be addressed, and accuracy enhanced to the degree technologically practical, to ensure a robust and adequate satellite on-orbit performance capability. This requires a continuing effort and sensitivity to the issues involved to provide economical LEO satellite systems and minimize the reboost requirements and design capability margins therefore.

\section{REFERENCES}

[1] K. O. Niehuss, H. C. Euler, and W. W. Vaughan, "Statistical technique for intermediate and long-range estimation of 13-month smoothed solar flux and geomagnetic index," NASA Tech. Rep. TM-4759, NASA Marshall Space Flight Center, Huntsville, AL, 1996.

[2] P. E. Dreher and A. T. Lyons, "Long-term orbital lifetime predictions," NASA Tech. Paper 3058, NASA Marshall Space Flight Center, Huntsville, AL, 1990.

[3] J. K. Owens, "NASA Marshall engineering thermosphere model-1999 version (MET-99)," NASA Tech. Memo., NASA Marshall Space Flight Center, Huntsville, AL, 2000, submitted for publication.

[4] Y. I. Vitinskii, "Solar activity forecasting," (in Russian), Israel Prog. Sci. Transl., Jerusalem, Israel, NASA TT F-289, National Aeronautics and Space Administration, Washington, DC, 1965.

[5] J. A. Joselyn, J. B. Anderson, H. C. Coffey, K. Harvey, D. Hathaway, G. Heckman, E. Hildner, W. Mende, K. Schatten, R. Thompson, A. W. P. Thompson, and O. R. White, "Panel achieves consensus prediction of solar cycle 23," Eos Trans. AGU, vol. 78, pp. 211-212, 1997.

[6] R. M. Wilson, "On the level of skill in predicting maximum sunspot number: A comparative study of single variate and bivariate precursor techniques," Solar Phys., vol. 125, pp. 143-155, 1990.

[7] K. Schatten, "Solar cycle 23 forecast update," Geophys. Res. Lett., vol. 25, pp. 599-601, 1998.

[8] A. G. McNish and J. V. Lincoln, "Prediction of sunspot numbers," Trans. Amer. Geophys. Union, vol. 30, p. 673, 1949.

[9] E. P. Boykin and T. J. Richards, "Application of the Lincoln-McNish technique to the prediction of the remainder of the twentieth sunspot cycle," Tech. Memo. 54/30-89, Lockheed Missiles and Space Company, Huntsville, AL, 1966

[10] R. L. Holland and W. W. Vaughan, "Lagrangian least-squares prediction of solar flux $\left(\mathrm{F}_{10.7}\right)$," J. Geophys. Res., vol. 89, pp. 11-16, 1984.

[11] W. W. Vaughan, J. K. Owens, K. O. Niehuss, and M. A. Shea, "The NASA Marshall solar activity model for use in predicting satellite drag," Adv. Space Res., vol. 23, pp. (4)715-(4)719, 1999.

[12] J. K. Owens, K. O. Niehuss, W. W. Vaughan, and M. A. Shea, "NASA Marshall engineering thermosphere model-1999 version (MET-99) and implications for satellite lifetime predictions," Adv. Space Res., vol. 26, pp. (1)157-(1)162, 2000.

[13] M. P. Hickey, "The NASA Marshall engineering thermosphere model," NASA CR-179359, 1988.

[14] _ " "An improvement in the integration procedure used in the NASA Marshall engineering thermosphere model," NASA CR-179389, 1988.

[15] F. A. Marcos, J. N. Bass, C. R. Baker, and W. S. Borer, "Neutral density models for aerospace applications," in 32nd Aerospace Sciences Meeting and Exhibit, 1994. AIAA Paper 94-0589.

[16] U. Von Zahn, "Mass spectrometric measurements of atomic oxygen in the upper atmosphere: A critical review," J. Geophys. Res., vol. 72, pp. 5933-5937, 1967.

[17] F. Marcos, M. Kendra, J. Griffin, J. Bass, J. Liu, and D. Larson, "Precision low earth orbit determination using atmospheric density calibration," Adv. Astron. Sci., vol. 97, pp. (1)515-(1)527, 1998.

[18] D. J. Gorney, "Solar cycle effects on the near-earth space environment," Rev. Geophys., vol. 28, pp. 315-336, 1990. 
Jerry K. Owens received the B.S. degree in physics from Tennessee Technological University in 1976, the M.S. degree in physics in 1986, and the M.S. degree in atmospheric science in 1996 from the University of Alabama, Huntsville. He is currently a Ph.D. candidate in atmospheric science at University of Alabama, Huntsville.

He currently serves as the Solar-B Project Systems Engineer in the Spaceflight Experiments Group of the Science Directorate at the Marshall Space Flight Center. He has over 25 years experience in space physics research, 15 of which are in upper atmospheric physics and space environments and effects. He develops, maintains, and updates models for estimation of thermospheric densities and future solar and geomagnetic activity, which provide inputs for spacecraft drag related calculations. He has served as Lead Instrument Scientist and/or Co-Investigator for experiments flown abroad several Space Shuttle and Spacelab missions. He also served as the Assistant Mission Scientist for the Astro-I Spacelab Mission from 1982 to 1984. His research interests and experience are varied, and they include aeronomy and space physics, analysis and modeling of space environment and spectroscopic data, spacecraft interactions with the space environment, and development of space flight instruments. He has authored or coauthored more than 40 papers in refereed journals, conference proceedings/presentations, and reports.

Mr. Owens is a Member of the American Institute of Aeronautics and Astronautics (AIAA) Atmospheric Environment Committee on Standards, the AIAA Atmospheric Environment Technical Committee, and the Space Physics and Aeronomy Section of the American Geophysical Union.

William W. Vaughan received the B.S. degree from University of Florida in 1951, the Grad. Cert. in atmospheric science from USAFIT (Florida State University) in 1952, and the Ph.D. degree in engineering-science from the University of Tennessee in 1975 .

$\mathrm{He}$ is Professor, Atmospheric Science at the University of Alabama, Huntsville. Previously, he was Director, Research Institute at the University. He was also Chief, Atmospheric Sciences Division and the Aerospace Environment Division at NASA Marshall Space Flight Center. He has over 45 years experience in atmospheric science research and the establishment and interpretation of aerospace environment requirements for the design of space vehicles and spacecraft. He is a professional member of the American Meteorological Society, American Institute of Aeronautics and Astronautics, American Geophysical Union, and American Society of Mechanical Engineers. His research has included atmospheric dynamics, reference atmospheres, solar activity, and climatological modeling. He has authored or coauthored over 100 technical reports, conference proceedings, and journal articles.
Keith O. Niehuss received the B.S. degree in aerospace engineering from Auburn University in Alabama.

$\mathrm{He}$ is a Senior Aerospace Engineer at Marshall Space Flight Center. He has 11 years experience in developing computer applications and software for application in various systems engineering activities. In particular, during the past several years his expertise was applied to thermospheric and solar activity models for application in estimating space environmental effects on spacecraft. He has also served as space environments and effects lead for tethered propulsion projects and provided space environments and affects definition support to a variety of space flight projects at the Marshall Space Flight Center. He served five years as the test engineer for the water processor of the environment control and life support equipment for the International Space Station. During this time, he developed control software and maintained electrical sensors for the test bed. He has authored or coauthored several technical reports, conference proceedings, and journal articles.

Joseph Minow received the B.A. degree in chemistry and biology from Western State College, CO, in 1981, the M.S. degree in physics from the University of Denver, CO, in 1987, and the Ph.D. degree in physics from the University of Alaska, Fairbanks, in 1997.

He is a Lead Engineer with Sverdrup Technology, Inc., Huntsville, AL, where he serves a Team Lead of Sverdrup's Environments Group that provides science and engineering support to Marshall Space Flight Center's Space Environments, Terrestrial and Planetary Environments, and Electromagnetic Environmental Effects Teams. His 17 years of experience in space and atmospheric physics include research in magnetospheric physics, auroral physics, airglow, and dynamics of the upper atmosphere as well as participation in ground-based optical studies of the aurora borealis at high-latitude sites in Alaska and Spitsbergen (arctic Norway). His current work is in the area of space environment interactions with spacecraft including the neutral atmosphere, plasma, and energetic particle environments. He has authored or coauthored more than 15 papers in refereed journals and conference proceedings.

Dr. Minow is a Member of the American Geophysical Union and the American Institute of Aeronautics and Astronautics. 